\title{
Efficiency Improvements of Antenna Optimization Using Orthogonal Fractional Experiments
}

\author{
Yen-Sheng Chen and Ting-Yu Ku \\ Department of Electronic Engineering, National Taipei University of Technology, 1 Sec. 3, Zhongxiao E. Road, Taipei 10608, Taiwan \\ Correspondence should be addressed to Yen-Sheng Chen; yschen@ntut.edu.tw
}

Received 22 June 2015; Revised 24 August 2015; Accepted 2 September 2015

Academic Editor: Toni Björninen

Copyright ( 2015 Y.-S. Chen and T.-Y. Ku. This is an open access article distributed under the Creative Commons Attribution License, which permits unrestricted use, distribution, and reproduction in any medium, provided the original work is properly cited.

\begin{abstract}
This paper presents an extremely efficient method for antenna design and optimization. Traditionally, antenna optimization relies on nature-inspired heuristic algorithms, which are time-consuming due to their blind-search nature. In contrast, design of experiments (DOE) uses a completely different framework from heuristic algorithms, reducing the design cycle by formulating the surrogates of a design problem. However, the number of required simulations grows exponentially if a full factorial design is used. In this paper, a much more efficient technique is presented to achieve substantial time savings. By using orthogonal fractional experiments, only a small subset of the full factorial design is required, yet the resultant response surface models are still effective. The capability of orthogonal fractional experiments is demonstrated through three examples, including two tag antennas for radiofrequency identification (RFID) applications and one internal antenna for long-term-evolution (LTE) handheld devices. In these examples, orthogonal fractional experiments greatly improve the efficiency of DOE, thereby facilitating the antenna design with less simulation runs.
\end{abstract}

\section{Introduction}

Following the rapid development of electromagnetic (EM) applications, it is of the utmost importance that the design cycle of antennas is reduced. Since antenna design problems usually involve a large number of design parameters, tuning the numerous unknowns by conventional trial-and-error approaches or one-factor-at-a-time parametric studies is very time-consuming. In order to cope with design problems more efficiently, nature-inspired heuristic algorithms have been successfully applied to many design instances. Among these optimizers, genetic algorithms (GAs) [1-4] and particle swarm optimization (PSO) [5-8] have drawn much more attention because of their simplicity and robustness. Also, other approaches including simulated annealing (SA) [9], ant colony optimization (ACO) [10], and invasive weed optimization (IWO) [11] have also been recognized as effective techniques for antenna design. However, heuristic algorithms usually need hundreds or even thousands of simulations due to their blind-search nature. No matter what the problem feature is, they just launch candidate solutions and search the solution domain by executing specific operators until a termination condition is met. Such a laborious process is so computationally intensive that the design cycle is inevitably expanded.

In opposition to the blind-search feature of these heuristic algorithms, a statistical optimization methodology called design of experiments (DOE) investigates the problem nature. DOE uses a predesigned simulation combination, analyzing the simulated results and building the response surface model of a design goal, which is the function of design parameters. In other words, the computational effort in DOE focuses on sequentially "building" the interested solution subdomain rather than iteratively "searching" the entire solution domain; therefore, it enables the design process to be more computationally efficient. DOE has been successfully applied to the EM design problems of flip-chip interconnects [12], microstrip vias [13], tag antennas for radio-frequency identification (RFID) [14], baluns [15], and annular slot ring antennas [16]. However, the above literature uses full factorial designs as the simulation combination. Although full factorial designs provide unbiased estimates on 
all the associated effects of design parameters, the number of required simulations grows exponentially as the number of design parameters increases. Therefore, it is often too costly to perform full factorial experiments. In these earlier reports, the number of design parameters is usually limited to not more than six to prevent hundreds of simulations; meanwhile, other parameters in the antenna structure are fixed at a certain level. If those fixed parameters are close to the true optimum value, these studies are still able to determine an optimum design having not more than six design parameters. However, real-world design problems usually involve a large number of unknowns. If prior knowledge of the fixed parameters is not included in the design process, the earlier approach may fail to find an optimum design, and this further lowers the usefulness of DOE.

In order to reduce the number of required simulations in DOE, this paper presents orthogonal fractional experiments as the simulation combination. The term "orthogonal" has two meanings. Firstly, in two-level experiments, all the column vectors in a simulation combination are perpendicular. That is, after normalizing the high level and low level of a parameter to +1 and -1 , respectively, any two column vectors in a treatment are orthogonal since their inner product is zero. Secondly, the term "orthogonal" in statistics is interpreted in the combinatorial sense. For any pair of columns in a treatment, all combinations of parameter levels occur at equal times. On the other hand, the term "fractional" comes from the fact that only a small fraction, or subset, of a "full" factorial design of experiment is performed. The purpose of this paper is to lend validity to orthogonal fractional experiments, which run only a small fraction of full factorial designs, yet the optimized results are impressing. The capability of orthogonal fractional experiments will be illustrated via three examples. The first design concerning an inductive-feed meander dipole is successfully developed by conducting merely 8 simulations. The second design regarding a complex dual-antenna system within a compact area of $0.1 \times 0.1 \lambda^{2}$ is achieved by 16 simulations. Finally, the third antenna structure having 12 design parameters for longterm-evolution (LTE) handheld applications is determined by simply 32 simulations. In these examples, the efficacy of orthogonal fractional experiments will be compared with full factorial designs, and the optimized performances will also be verified by antenna measurements.

\section{Review of DOE}

Before the achievement of orthogonal fractional experiments is detailed, this section reviews the procedure of DOE. In particular, the difference between full factorial designs and orthogonal fractional experiments will be thoroughly discussed.

DOE considers a design problem as a process, shown in Figure 1. The output responses $y_{1}, y_{2}, \ldots, y_{n}$ are usually the design goals of an antenna, and the input factors are design parameters $x_{1}, x_{2}, \ldots, x_{k}$. To quantify how influential the design parameters are, antenna designers purposefully vary them at two levels. The degree of a parameter's individual

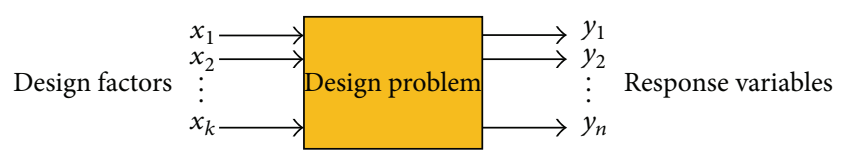

FIGURE 1: General model of the DOE technique.

influence on a response variable is called the main effect $E_{i}$ of the parameter $x_{i}$. Usually, the main effect $E_{i}$ of $x_{i}$ does not hold constant when other parameters alter their levels. The influence of $x_{j}(j \neq i)$ on $E_{i}$ is called the two-factor interaction $E_{i j}$ between them. Likewise, two-factor interaction $E_{i j}$ between $x_{i}$ and $x_{j}$ may change when the other parameter $x_{l}$ $(l \neq j \neq i)$ is involved, and the resultant influence is called the three-factor $E_{i j l}$ between them. Similarly, other higher-order interactions between multiple factors can thus be defined. Estimating these effects as precisely as possible is the major computational effort in DOE.

2.1. Full Factorial Designs. After the two levels of $k$ design parameters are assigned, the complete arrangements result in a combination of $2^{k}$ simulations; this experimental strategy is called a $2^{k}$ full factorial design. The $2^{k}$ full factorial design constructs a response surface model expressed as

$$
\begin{aligned}
\widehat{y}= & \beta_{0}+\sum_{i=1}^{k} \beta_{i} x_{i}+\sum_{i=1}^{k-1} \sum_{j=i+1}^{k} \beta_{i j} x_{i} x_{j} \\
& +\sum_{i=1}^{k-2} \sum_{j=i+1}^{k-1} \sum_{l=j+1}^{k} \beta_{i j l} x_{i} x_{j} x_{j}+\cdots+\beta_{i j \cdots k} x_{i} x_{j} \cdots x_{k},
\end{aligned}
$$

where $\hat{y}$ is the predicted value of an antenna response variable, $x_{i}(i=1,2, \ldots, k)$ is the design parameter normalized from its high level and low level to +1 and -1 , respectively, $\beta_{i}$ is the coefficient proportional to the main effect $E_{i}$, and $\beta_{i j}$ is the coefficient proportional to the two-factor interaction $E_{i j}$, and the coefficients for other higher-order interactions are thus denoted. These coefficients are the unknowns to be estimated from the experimental results.

Model (1) is called a saturated model; it contains all possible interactions, up to and including the $k$-factor interaction. The major benefit of $2^{k}$ full factorial designs is that they provide detailed information on all the associated effects. Besides, they are relatively easy to implement. However, although (1) is a thorough expression, three disadvantages are observed. Firstly, the model involves so many higherorder terms that the complexity of succeeding optimization arises. Secondly, a model with higher-order interactions is more difficult to interpret. Thirdly and most importantly, a large number of unknown coefficients are cast in this model. The estimation of all the coefficients will be very inefficient as $k$ increases, for the number of required simulations grows exponentially.

2.2. Orthogonal Fractional Experiments. In order to reduce the number of required simulations, this paper recommends using orthogonal fractional factorial design of experiments 
for complex antenna development. Generally, an antenna design problem has a hierarchical property; that is, when a large number of design parameters are involved, an antenna response is primarily driven by some of the main effects and lower-order interactions. A higher-order interaction is significant only if all lower-order terms involving subsets of the parameters in that term are included. In such situations, the following two-factor interaction model for $2^{k}$ factorial is very useful:

$$
\widehat{y}=\beta_{0}+\sum_{i=1}^{k} \beta_{i} x_{i}+\sum_{i=1}^{k-1} \sum_{j=i+1}^{k} \beta_{i j} x_{i} x_{j},
$$

where $\beta_{i}$ and $\beta_{i j}$ are again the unknown coefficients proportional to the main effect and two-factor interactions, respectively. Since higher-order interactions are excluded, (2) is more convenient for interpretation and optimization. More importantly, the number of unknown coefficients drops, so the number of required simulations decreases. In fact, this is what orthogonal fractional experiments focus on. By performing a small fraction of full factorial design, orthogonal fractional experiments provide unbiased estimates on those main effects and two-factor interactions, so a useful model can be attained in a more efficient way.

To construct a $2^{k-p}$ orthogonal fractional experiment, we first create a full factorial design for $k$ - $p$ parameters. These $k$ - $p$ parameters are denoted as the basic variables, and the setting of the remaining $p$ parameters, called additional variables, is assigned by multiplying together the coded states of the basic variables. The kernel of orthogonal fractional experiments lies in how to assign these multiplicators, called the design generator. The guideline for the construction of design generator begins by enumerating all the possible design generators for a given $k$ and $p$. Next, the defining relation of a design generator can be determined by expressing all the $C_{2}^{p}+C_{3}^{p}+\cdots+C_{p}^{p}=2^{p}-p-1$ combinations of design generator's multiplication. Therefore, each design generator has its own defining relation, and the rank of all the possible design generators is further sorted by an index called "resolution." By defining, the resolution is the length of the shortest term in the defining relation. The larger the resolution, the better the design generator. Consequently, every design generator is mapped to an associated level of resolution, so a designer is able to determine the best design generator based on the resolution for a given $k$ and $p$. Another implication of resolution is that it indicates how main effects and interactions are aliasing. That is, a design of resolution $R$ has no $n$-factor effect confounded with any other effect containing less than $R-n$ factors.

Once the simulation combination is planned by either full factorial designs or orthogonal fractional experiments, the response of design goals can be simulated, and the DOE procedure can proceed to the stage of experimental analysis.

2.3. Analysis of Experiments. Those simulated results provide useful information about the design problem. In particular, the main effect $E_{i}$ is estimated by subtracting the average of simulated results for $x_{i}$ fixed at high level $\left(x_{i}=+1\right)$ from the average of simulated results for $x_{i}$ fixed at low level $\left(x_{i}=-1\right)$. The two-factor interaction $E_{i j}$ between $x_{i}$ and $x_{j}$ is estimated by subtracting the average main effect $E_{i}$ for the parameter $x_{j}$ fixed at high level from the average $E_{i}$ for $x_{j}$ fixed at low level. Likewise, three-factor interaction $E_{i j l}$ between $x_{i}, x_{j}$, and $x_{l}$ is estimated by the average $E_{i j}$ for $x_{l}$ fixed at high level from the average $E_{i j}$ for $x_{l}$ fixed at low level. By similar approaches, other higher-order interactions are thus defined and estimated. After computing these effects, the statistical significance of them is further inferred by repeated analysis of variance (ANOVA) with a stepwise selection procedure [17]. The operation begins with a forward selection procedure, which selects a response surface model beginning from the most significant effect and adding the terms selected from the most significant effect one by one until a certain level of significance for the model is reached. Then, the operation continues with a backward elimination, excluding one by one the smallest effects from the resultant model of forward selection until another level of significance is reached. The forward selection and backward elimination procedures are repeated, until none of the effects should be added to or eliminated from the model.

Finally, the estimated coefficients of response surface models are computed by dividing the associated effects by two. This is because the coefficients in models (1) and (2) account for a one-unit increase, whereas the estimated effects specify a two-unit increase varied from -1 to +1 . In addition, the intercept of the models is estimated by the average of all the simulated results. Consequently, with the mathematical models at hand, numerical optimization methods can be applied to these surrogates straightforwardly, and the optimum design is thus determined.

In order to validate the capability of orthogonal fractional experiments, three design problems are considered: (1) an inductive-feed meander dipole for passive tags, (2) a complex dual-antenna structure for passive tags, and (3) an internal antenna for LTE handheld devices.

\section{An Inductive-Feed Meander Dipole for Passive Tags}

The design of tag antennas for passive RFID systems is particularly difficult because the impedance matching does not aim at 50- $\Omega$ transmission lines. The design goal is to make the antenna impedance $\left(R_{a}+j X_{a}\right)$ conjugate match to the appended chip impedance, which is usually a highly capacitive reactance. To compensate the large capacitive reactance, the inductive-feed structure shown in Figure 2 is a popular choice [18]. Thus, this benchmarking structure is utilized to study the validity of orthogonal fractional experiments. It consists of a meander dipole antenna and a coupled rectangular loop, to which the chip will be mounted, printed on a $0.8-\mathrm{mm}$ thick FR4 (dielectric constant $\varepsilon_{r}=4.4$ and loss tangent $\tan \delta=0.02$ ). In this work, an Impinj Monza Gen2 tag chip with a complex impedance 33 - j112 $\Omega$ [19] at $915 \mathrm{MHz}$ is chosen as an example; therefore, the design goals are $R_{a}=33 \Omega$ and $X_{a}=112 \Omega$ at $915 \mathrm{MHz}$. The design parameters include the length of the feeding loop $b$, 
TABLE 1: Ranges for the design parameter of the inductive-feed meander dipole.

\begin{tabular}{lcc}
\hline Parameter & Low level $(-1)$ & High level $(+1)$ \\
\hline$b$ & $7 \mathrm{~mm}$ & $12 \mathrm{~mm}$ \\
$a$ & $7 \mathrm{~mm}$ & $12 \mathrm{~mm}$ \\
$d$ & $0.2 \mathrm{~mm}$ & $2.8 \mathrm{~mm}$ \\
$l$ & $10 \mathrm{~mm}$ & $14 \mathrm{~mm}$ \\
$t$ & $5 \mathrm{~mm}$ & $8 \mathrm{~mm}$ \\
\hline
\end{tabular}

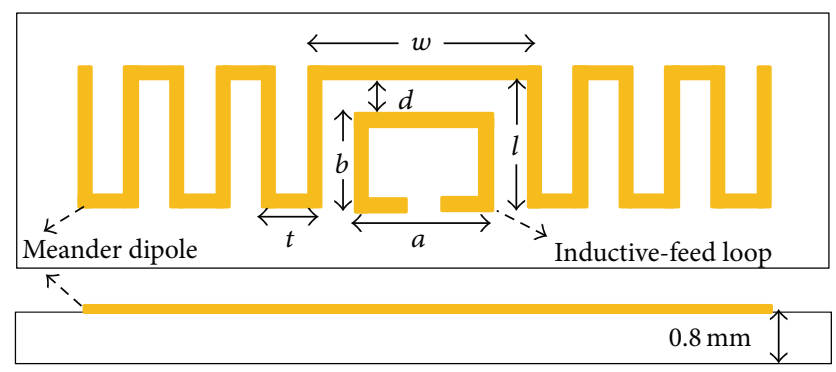

Figure 2: The benchmarking configuration of the inductive-feed meander dipole.

the width of the feeding loop $a$, the gap between the loop and the mender dipole $d$, the length of the central segment $w$, the length of the transverse segment $l$, and the distance between adjacent transverse segments $t$. In this case, $w$ is fixed at $35 \mathrm{~mm}$ due to a size constraint. Since UHF RFID tags aim at item-level tagging, the size of a tag antenna cannot be too large. Therefore, the benchmarking structure is highly meandered so that the antenna has a compact size. Considering such a meander structure, the most important parameters are the segments which are meandered, namely, $l$ and $t$, so they are selected as the design parameters. On the other hand, the length of the segment which is not meandered, namely, $w$, should be kept at a certain small level so that the dimension of the tag antenna is electrically small. Therefore, $w$ is not assigned as a design parameter, and it is fixed at $35 \mathrm{~mm}$. If the size constraint is regardless, $w$ can also be assigned as a design parameter, and the proposed design technique is generally applicable. Each of the remaining five design parameters, namely, $b, a, d, l$, and $t$, is varied at two levels, respectively. The chosen ranges are shown in Table 1.

3.1. Full Factorial Designs. The first case to be investigated is the $2^{5}$ full factorial design, which provides the greatest information but spends the hugest computational effort. Table 2 shows the simulation combination conducted in the experiment and the associated simulated results by Ansoft HFSS. From these results, all the 32 information items, including five main effects, 26 multiple-factor interactions, and one mean response, can be estimated. After that, the significance of these effects is examined by the repeated ANOVA. The resultant response surface models are expressed as

$$
\begin{aligned}
\widehat{R}_{a}(\Omega)= & 10.49+3.00\left(\frac{b-9.5}{2.5}\right)+5.76\left(\frac{a-9.5}{2.5}\right) \\
& -5.50\left(\frac{d-1.5}{1.3}\right)-2.91\left(\frac{t-6.5}{1.5}\right)
\end{aligned}
$$

TABLE 2: The $2^{5}$ full factorial design and the simulated results (at $915 \mathrm{MHz}$ ).

\begin{tabular}{lccccccc}
\hline Run & $b$ & $a$ & $d$ & $l$ & $t$ & $R_{a}(\Omega)$ & $X_{a}(\Omega)$ \\
\hline 1 & -1 & -1 & -1 & -1 & -1 & 6.94 & 74.41 \\
2 & +1 & -1 & -1 & -1 & -1 & 12.8 & 123.16 \\
3 & -1 & +1 & -1 & -1 & -1 & 25.29 & 117.67 \\
4 & +1 & +1 & -1 & -1 & -1 & 43.13 & 177.08 \\
5 & -1 & -1 & +1 & -1 & -1 & 1.77 & 75.21 \\
6 & +1 & -1 & +1 & -1 & -1 & 4.02 & 125.36 \\
7 & -1 & +1 & +1 & -1 & -1 & 6.34 & 123.11 \\
8 & +1 & +1 & +1 & -1 & -1 & 14.91 & 194.62 \\
9 & -1 & -1 & -1 & +1 & -1 & 6.46 & 62.86 \\
10 & +1 & -1 & -1 & +1 & -1 & 11.63 & 103.33 \\
11 & -1 & +1 & -1 & +1 & -1 & 22.87 & 104.79 \\
12 & +1 & +1 & -1 & +1 & -1 & 33.88 & 139.29 \\
13 & -1 & -1 & +1 & +1 & -1 & 1.67 & 63.66 \\
14 & +1 & -1 & +1 & +1 & -1 & 3.68 & 104.28 \\
15 & -1 & +1 & +1 & +1 & -1 & 5.92 & 103.03 \\
16 & +1 & +1 & +1 & +1 & -1 & 12.98 & 157.83 \\
17 & -1 & -1 & -1 & -1 & +1 & 3.82 & 56.99 \\
18 & +1 & -1 & -1 & -1 & +1 & 6.86 & 93.47 \\
19 & -1 & +1 & -1 & -1 & +1 & 13.83 & 92.93 \\
20 & +1 & +1 & -1 & -1 & +1 & 23.55 & 135.52 \\
21 & -1 & -1 & +1 & -1 & +1 & 1 & 57.86 \\
22 & +1 & -1 & +1 & -1 & +1 & 2.24 & 94.2 \\
23 & -1 & +1 & +1 & -1 & +1 & 3.59 & 93.26 \\
24 & +1 & +1 & +1 & -1 & +1 & 8.08 & 142.22 \\
25 & -1 & -1 & -1 & +1 & +1 & 3.54 & 50.64 \\
26 & +1 & -1 & -1 & +1 & +1 & 6.27 & 82.1 \\
27 & -1 & +1 & -1 & +1 & +1 & 12.58 & 81.94 \\
28 & +1 & +1 & -1 & +1 & +1 & 22.4 & 119.46 \\
29 & -1 & -1 & +1 & +1 & +1 & 0.92 & 50.77 \\
30 & +1 & -1 & +1 & +1 & +1 & 2.06 & 82.46 \\
31 & -1 & +1 & +1 & +1 & +1 & 3.31 & 81.59 \\
32 & +1 & +1 & +1 & +1 & +1 & 7.25 & 122.24 \\
\hline & & & & & & &
\end{tabular}

$$
\begin{aligned}
& +1.53\left(\frac{b-9.5}{2.5}\right)\left(\frac{a-9.5}{2.5}\right) \\
& -1.08\left(\frac{b-9.5}{2.5}\right)\left(\frac{d-1.5}{1.3}\right) \\
& -2.94\left(\frac{a-9.5}{2.5}\right)\left(\frac{d-1.5}{1.3}\right) \\
& -1.51\left(\frac{a-9.5}{2.5}\right)\left(\frac{t-6.5}{1.5}\right) \\
& +1.48\left(\frac{d-1.5}{1.3}\right)\left(\frac{t-6.5}{1.5}\right) \\
\widehat{X}_{a}(\Omega)= & 102.73+22.06\left(\frac{b-9.5}{2.5}\right) \\
& +21.43\left(\frac{a-9.5}{2.5}\right)-8.34\left(\frac{l-12}{2}\right)
\end{aligned}
$$


TABLE 3: The $2^{5-2}$ orthogonal fractional experiment and the simulated results (at $915 \mathrm{MHz}$ ).

\begin{tabular}{lccccccc}
\hline Run & $b$ & $a$ & $d$ & $l$ & $t$ & $R_{a}(\Omega)$ & $X_{a}(\Omega)$ \\
\hline 1 & -1 & -1 & -1 & +1 & +1 & 3.54 & 50.64 \\
2 & +1 & -1 & -1 & -1 & -1 & 12.8 & 123.16 \\
3 & -1 & +1 & -1 & -1 & +1 & 13.83 & 92.93 \\
4 & +1 & +1 & -1 & +1 & -1 & 33.88 & 139.29 \\
5 & -1 & -1 & +1 & +1 & -1 & 1.67 & 63.66 \\
6 & +1 & -1 & +1 & -1 & +1 & 2.24 & 94.2 \\
7 & -1 & +1 & +1 & -1 & -1 & 6.34 & 123.11 \\
8 & +1 & +1 & +1 & +1 & +1 & 7.25 & 122.24 \\
\hline
\end{tabular}

$$
\begin{aligned}
& -12.88\left(\frac{t-6.5}{1.5}\right) \\
& -2.95\left(\frac{b-9.5}{2.5}\right)\left(\frac{t-6.5}{1.5}\right) .
\end{aligned}
$$

With these models functioning as surrogates, numerical optimization methods can be applied to these expressions to have $R_{a}=33 \Omega$ and $X_{a}=112 \Omega$. The optimum design is $b=7.85 \mathrm{~mm}, a=12.0 \mathrm{~mm}, d=0.28 \mathrm{~mm}, l=13.52 \mathrm{~mm}$, and $t=5.02 \mathrm{~mm}$. This design is readily verified by HFSS, and the simulated results are $R_{a}=26.84 \Omega$ and $X_{a}=106.11 \Omega$ at $915 \mathrm{MHz}$.

3.2. Orthogonal Fractional Experiments. Next, the efficiency of the proposed method, namely, orthogonal fractional experiments, is verified. To perform the minimum number of simulations yet to predict the antenna responses as precisely as possible, a resolution III $2^{5-2}$ fractional design is employed. The process starts by choosing $b, a$, and $d$ as the basic variables, constructing its associated $2^{3}$ full factorial design. The state of the two additional variables, namely, $l$ and $t$, is assigned as $l=b \times a$ and $t=b \times d$, respectively. The resultant 8 simulation combinations and the corresponding results are shown in Table 3.

Before estimating the coefficients in the response surface models, it is important to investigate the confounding pattern of all effects. The entire defining relation can be deduced by multiplying the design generators together; namely, $I=b \times a \times$ $l=b \times d \times t=a \times d \times l \times t$, where $I$ denotes the identity column. In the identity column, all the levels are always +1 . Afterward, the complex aliasing pattern among effects can be determined by using the defining relation. Multiplying any column (or effect) by defining relation obtains the aliases for that column (or effect). For example, the alias of the main effect of $a$ can be attained by

$$
\begin{aligned}
(a) \times I & =(a) \times b \times a \times l=(a) \times b \times d \times t \\
& =(a) \times a \times d \times l \times t .
\end{aligned}
$$

Because the square of any column is just the identity $I$, the aliasing pattern of the main effect of $a$ is

$$
a+b * l+a * b * d * t+d * l * t
$$

where the symbol “*” denotes the multiple-factor interaction resulting from the associated parameters. For example, $b * l$ represents the two-factor interaction of $b$ and $l$, and $a * b *$ $d * t$ represents the four-factor interaction of $a, b, d$, and $t$. In addition, $a+b * l+a * b * d * t+d * l * t$ indicates that the main effect of $a$, the two-factor interaction of $b$ and $l$, the four-factor interaction of $a, b, d$, and $t$, and the three-factor interaction of $d, l$, and $t$ are confounded together. Similarly, other confounding patterns can be identified as

$$
\begin{gathered}
b+a * l+d * t+a * b * d * l * t \\
d+b * a * d * l+b * t+a * l * t \\
l+b * a+b * d * l * t+a * d * t \\
t+b * a * l * t+b * d+a * d * l \\
a * d+b * d * l+b * a * t+l * t \\
a * t+b * l * t+b * a * d+d * l \\
I+b * a * l+b * d * t+a * d * l * t .
\end{gathered}
$$

Although the estimated effects are accompanied by some higher-order interactions, based on the sparsity-of-effects principle, only the main effects are cast into the significant test. The response surface models with significant coefficients are

$$
\begin{aligned}
\widehat{R}_{a}(\Omega)= & 10.20+3.85\left(\frac{b-9.5}{2.5}\right)+5.13\left(\frac{a-9.5}{2.5}\right) \\
& -5.82\left(\frac{d-1.5}{1.3}\right)-3.48\left(\frac{t-6.5}{1.5}\right), \\
\widehat{X}_{a}(\Omega)= & 101.16+18.57\left(\frac{b-9.5}{2.5}\right) \\
& +18.24\left(\frac{a-9.5}{2.5}\right)-7.20\left(\frac{l-12}{2}\right) \\
& -11.15\left(\frac{t-6.5}{1.5}\right) .
\end{aligned}
$$

It is noted that the models obtained by orthogonal fractional experiments are much simpler as compared to those determined by full factorial designs. Afterward, numerical optimization is applied to these surrogates, and the associated parameters of the optimum solution are $b=8.95 \mathrm{~mm}, a=$ $11.95 \mathrm{~mm}, d=0.2 \mathrm{~mm}, l=13.76 \mathrm{~mm}$, and $t=5 \mathrm{~mm}$. The verified results by HFSS are $R_{a}=27.54 \Omega$ and $X_{a}=112.54 \Omega$. Clearly, even though the response surfaces are built by merely 8 designed simulations, the optimum design still meets our specification.

In order to compare the detailed performances between full factorial designs and orthogonal fractional experiments, the power reflection coefficients, which have been normalized to $33-j 112 \Omega$, are shown in Figure 3. These results clearly show that the performances are impressively excellent no matter which experimental design is used. However, the technique of orthogonal fractional experiments, which only costs 8 simulations, greatly improves the optimization efficiency. 


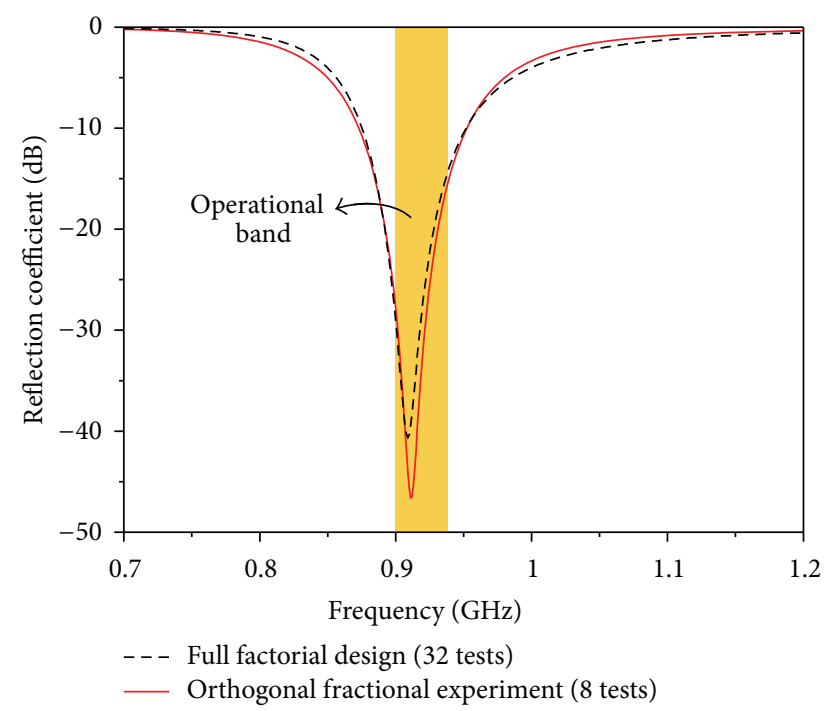

FIGURE 3: Comparison of the optimized inductive-feed meander dipoles between the full factorial design and the orthogonal fractional experiment.

In contrast, full factorial designs require 32 simulations, yet the optimized performances are not better than those determined by orthogonal fractional experiments. In other words, the proposed method reduces the design cycle by $75 \%$.

\section{A Dual-Antenna Structure for Passive Tags}

Next, let us examine orthogonal fractional experiments by a more complicated design problem. The antenna to be investigated is a dual-antenna structure (see Figure 4), which is developed to overcome the two inherent limitations of traditional passive tag structures. The first one is that when a single antenna is used for both power reception and signal backscattering, there would be no power received by the chip as the tag antenna is switched to the short state of loading impedance. Secondly, the two impedance states, namely, the short and conjugate matched states, would not provide the maximum level difference in the backscattered signals, thereby resulting in a shorter read range. Fortunately, a dual-antenna structure for passive RFID tags overcomes the two limitations simultaneously [20]. The dual-antenna structure consists of two independent antennas, one exclusively for receiving and the other for backscattering, such that the requirements for both continuous power supply and maximum level difference in the antenna backscattering can be achieved. However, such a complex antenna system has several design goals which must be attained simultaneously so that its performance will be optimized. These design goals include that the input impedance of the receiving antenna $\left(R_{\mathrm{re}}+j X_{\mathrm{re}}\right)$ should be conjugate matched to that of the tag chip, the input reactance of the backscattering antenna $\left(X_{\mathrm{sc}}\right)$ must be zero, and the mutual coupling $\left(\left|S_{21}\right|\right)$ between the two antennas should be minimized.

The benchmarking topology shown in Figure 4 consists of two linearly tapered meander dipoles printed on the opposite

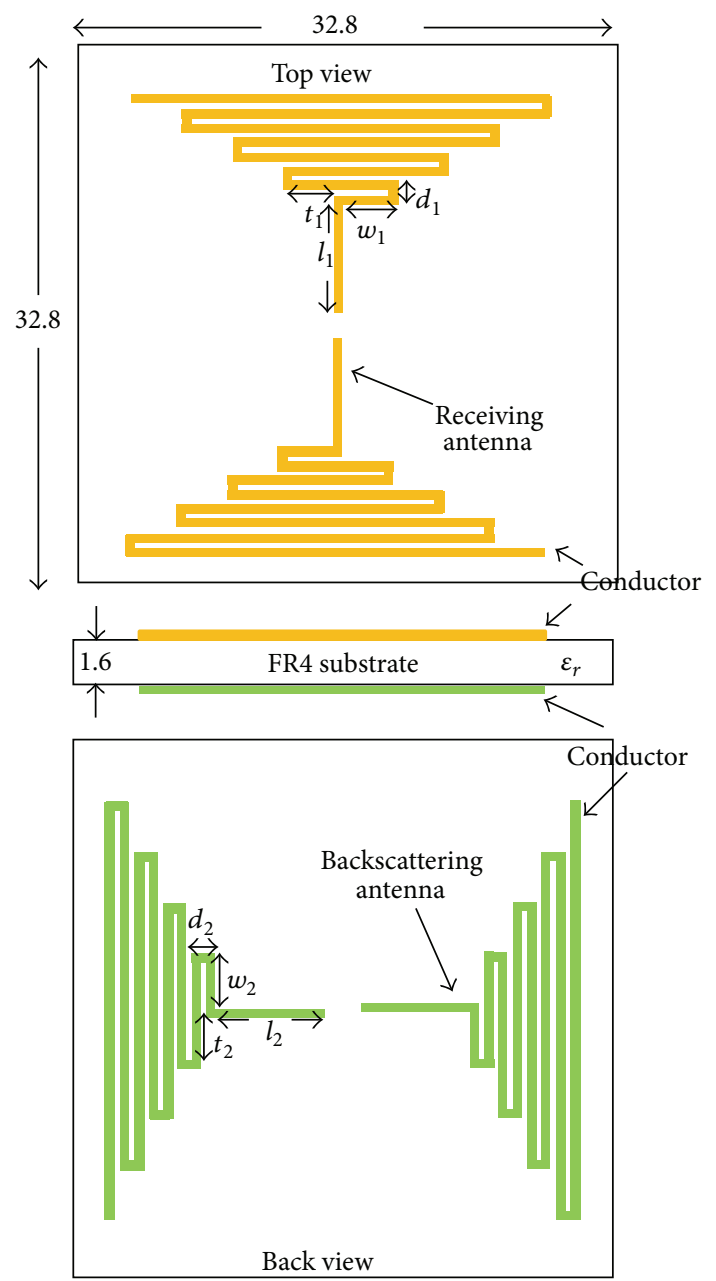

FIGURE 4: The benchmarking configuration of the dual-antenna structure.

sides of a 1.6-mm thick FR4 substrate. The two antennas are perpendicular to each other, and both antennas are antisymmetric and of uniform strip width of $0.2 \mathrm{~mm}$. The design parameters for the receiving (backscattering) antenna include the length of the central segments $l_{1}\left(l_{2}\right)$, the length of the first transverse segments $w_{1}\left(w_{2}\right)$, the spacing between adjacent transverse segments $d_{1}\left(d_{2}\right)$, and the uniform increment in length $t_{1}\left(t_{2}\right)$. Furthermore, the design space is set to be $0.1 \times 0.1 \lambda^{2}$, namely, $32.8 \times 32.8 \mathrm{~mm}^{2}$ at $915 \mathrm{MHz}$, suitable for tagging most small objects in UHF RFID applications. Likewise, the chip impedance is set to be $33-j 112 \Omega$ at $915 \mathrm{MHz}$.

In this example, the selected response variables are the input resistance and reactance of the receiving antenna, the input reactance of the backscattering antenna, and the isolation between them, namely, $R_{\mathrm{re}}, X_{\mathrm{re}}, X_{\mathrm{sc}}$, and $\left|S_{21}\right|$, all at the central frequency of the operational band (902$928 \mathrm{MHz}$ ). The design goals are, at $915 \mathrm{MHz}, R_{\mathrm{re}}=33 \Omega$, $X_{\mathrm{re}}=112 \Omega, X_{\mathrm{sc}}=0 \Omega$, and a minimized $\left|S_{21}\right|$. Although there are totally 8 design parameters indicated in Figure 4, it is found that the effects of the lengths of the central segments $l_{1}$ and $l_{2}$ on the response variables are insignificant because 
TABLE 4: Ranges for the design parameter of the dual-antenna structure.

\begin{tabular}{lcc}
\hline Parameter & Low level $(-1)$ & High level $(+1)$ \\
\hline$w_{1}$ & $3.5 \mathrm{~mm}$ & $4 \mathrm{~mm}$ \\
$d_{1}$ & $0.8 \mathrm{~mm}$ & $1.2 \mathrm{~mm}$ \\
$t_{1}$ & $3 \mathrm{~mm}$ & $3.5 \mathrm{~mm}$ \\
$w_{2}$ & $2.5 \mathrm{~mm}$ & $3 \mathrm{~mm}$ \\
$d_{2}$ & $0.8 \mathrm{~mm}$ & $1.2 \mathrm{~mm}$ \\
$t_{2}$ & $2.75 \mathrm{~mm}$ & $3.25 \mathrm{~mm}$ \\
\hline
\end{tabular}

of the size constraint of the antenna structure. Due to the requirement of item-level tagging, the antenna size is set to $0.1 \times 0.1 \lambda^{2}$. Within such an electrically small area, the range of $l_{1}$ and $l_{2}$ in the DOE process is very limited, so moving these parameters from the low level to the high level causes insignificant change on the response variables. As a result, their effects are found to be statistically insignificant, and their values are both fixed at $7 \mathrm{~mm}$. The ranges of the other parameters are listed in Table 4.

4.1. Full Factorial Designs. Firstly, the $2^{6}$ full factorial design is considered. The simulation combination and the associated simulated results are shown in Table 5. Once the 64 simulations have been conducted, all the main effects and the interactions on each response variable can be estimated. Afterward, the statistical significances of these effects are examined by the repeated ANOVA, and the resultant models for $R_{\mathrm{re}}, X_{\mathrm{re}}, X_{\mathrm{sc}}$, and $\left|S_{21}\right|$ at $915 \mathrm{MHz}$ are thus expressed as

$$
\begin{aligned}
\widehat{R}_{\mathrm{re}} & (\Omega)=15.33+1.22\left(\frac{w_{1}-3.75}{0.25}\right) \\
+ & 4.79\left(\frac{t_{1}-3.25}{0.25}\right)-0.33\left(\frac{d_{2}-1}{0.2}\right) \\
+ & 0.71\left(\frac{w_{1}-3.75}{0.25}\right)\left(\frac{t_{1}-3.25}{0.25}\right)-0.25\left(\frac{d_{1}-1}{0.2}\right) \\
& \left(\frac{t_{2}-3}{0.25}\right), \\
\widehat{X}_{\mathrm{re}} & (\Omega)=151.38+24.39\left(\frac{w_{1}-3.75}{0.25}\right) \\
& +7.17\left(\frac{d_{1}-1}{0.2}\right)+106.53\left(\frac{t_{1}-3.25}{0.25}\right) \\
& -14.33\left(\frac{d_{2}-1}{0.2}\right)+9.76\left(\frac{t_{2}-3}{0.25}\right) \\
& +7.46\left(\frac{w_{1}-3.75}{0.25}\right)\left(\frac{t_{1}-3.25}{0.25}\right)-5.25\left(\frac{d_{1}-1}{0.2}\right) \\
& +\left(\frac{t_{2}-3}{0.25}\right)+3.48\left(\frac{t_{1}-3.25}{0.25}\right)\left(\frac{t_{2}-3}{0.25}\right) \\
& -4.2\left(\frac{d_{2}-1}{0.2}\right)\left(\frac{t_{2}-3}{0.25}\right),
\end{aligned}
$$

$$
\begin{aligned}
& \widehat{X}_{\mathrm{sc}}(\Omega)=-3.56-12.31\left(\frac{d_{1}-1}{0.2}\right) \\
& +6.18\left(\frac{t_{1}-3.25}{0.25}\right)+7.17\left(\frac{d_{2}-1}{0.2}\right) \\
& +71.48\left(\frac{t_{2}-3}{0.25}\right)+16.37\left(\frac{w_{2}-2.75}{0.25}\right) \\
& -3\left(\frac{d_{1}-1}{0.2}\right)\left(\frac{t_{1}-3.25}{0.25}\right)+3.3\left(\frac{w_{2}-2.75}{0.25}\right) \\
& \cdot\left(\frac{t_{2}-3}{0.25}\right) \\
& \left|\widehat{S}_{21}\right|(\mathrm{dB})=-37.39-1.62\left(\frac{d_{1}-1}{0.2}\right) \\
& -5.7\left(\frac{t_{1}-3.25}{0.25}\right)+1.88\left(\frac{t_{2}-3}{0.25}\right) \\
& -2.93\left(\frac{w_{2}-2.75}{0.25}\right)+1.34\left(\frac{d_{1}-1}{0.2}\right)\left(\frac{t_{2}-3}{0.25}\right) \\
& +1.3\left(\frac{t_{1}-3.25}{0.25}\right)\left(\frac{w_{2}-2.75}{0.25}\right)-2\left(\frac{t_{2}-3}{0.25}\right) \\
& \cdot\left(\frac{w_{2}-2.75}{0.25}\right)-6.59\left(\frac{t_{1}-3.25}{0.25}\right)\left(\frac{t_{2}-3}{0.25}\right) \\
& -3.83\left(\frac{t_{1}-3.25}{0.25}\right)\left(\frac{t_{2}-3}{0.25}\right)\left(\frac{w_{2}-2.75}{0.25}\right) \\
& +1.42\left(\frac{t_{1}-3.25}{0.25}\right)\left(\frac{d_{2}-1}{0.2}\right)\left(\frac{t_{2}-3}{0.25}\right)\left(\frac{w_{2}-2.75}{0.25}\right) \\
& -1.25\left(\frac{w_{1}-3.75}{0.25}\right)\left(\frac{t_{1}-3.25}{0.25}\right)\left(\frac{d_{2}-1}{0.2}\right)\left(\frac{t_{2}-3}{0.25}\right) \\
& \cdot\left(\frac{w_{2}-2.75}{0.25}\right)
\end{aligned}
$$

Having the four response surface models, the optimum design can be attained by solving the constrained optimization problem, in which $\left|S_{21}\right|$ should be minimized subject to the constraints of $R_{\mathrm{re}} \geq 12,102 \leq X_{\mathrm{re}} \leq 122$, and $-10 \leq$ $X_{\mathrm{sc}} \leq 10$. The parameters of the optimum dual-antenna design are $w_{1}=3.5 \mathrm{~mm}, d_{1}=0.91 \mathrm{~mm}, t_{1}=3.19 \mathrm{~mm}, w_{2}=$ $3 \mathrm{~mm}, d_{2}=0.8 \mathrm{~mm}$, and $t_{2}=2.97 \mathrm{~mm}$. For verification, this design is simulated by HFSS, and the obtained responses at $915 \mathrm{MHz}$ are $R_{\mathrm{re}}=12.75 \Omega, X_{\mathrm{re}}=113.78 \Omega, X_{\mathrm{sc}}=-3.57 \Omega$, and $\left|S_{21}\right|=-48.4 \mathrm{~dB}$. Clearly, the design goals are achieved by performing 64 simulations.

4.2. Orthogonal Fractional Experiments. In order to reduce the number of tests, a resolution IV $2^{6-2}$ orthogonal fractional experiment is demonstrated. The proposed $2^{6-2}$ experiment begins with a basic design, which consists of 16 runs associated with a full factorial in $w_{1}, d_{1}, t_{1}$, and $d_{2}$. Then, two additional columns for $t_{2}$ and $w_{2}$ are added by $t_{2}=w_{1} \times d_{1} \times t_{1}$ and $w_{2}=w_{1} \times d_{2} \times t_{2}$, respectively. Therefore, the complete 
TABLE 5: The $2^{6}$ full factorial design and the simulated results (at $915 \mathrm{MHz})$.

\begin{tabular}{|c|c|c|c|c|c|c|c|c|c|c|}
\hline un & & $d_{1}$ & $t_{1}$ & $d$ & $t_{2}$ & $w_{2}$ & $R_{\mathrm{re}}(\Omega)$ & $X_{\mathrm{re}}(\Omega)$ & $\Lambda_{\mathrm{sc}}(\Omega L)$ & $21 / \mathrm{Cut}_{2}$ \\
\hline & -1 & -1 & -1 & -1 & -1 & & 10.18 & 20.64 & -96.97 & -32.56 \\
\hline & +1 & & -1 & - & -1 & & 11.00 & 49.51 & 0075 & -29.09 \\
\hline & -1 & +1 & -1 & -1 & -1 & & 10.27 & 35.98 & -111.41 & -35.76 \\
\hline & & +1 & -1 & -1 & -1 & -1 & 11.58 & 77.42 & -107.85 & -35.72 \\
\hline & -1 & & +1 & & -1 & -1 & 18.18 & 213.59 & -84.09 & -41.58 \\
\hline & +1 & -1 & +1 & -1 & -1 & -1 & 21.41 & 269.02 & -77.7 & -43.84 \\
\hline & -1 & +1 & +1 & -1 & -1 & -1 & 18.66 & 231.22 & -112.58 & -46.48 \\
\hline & & +1 & +1 & -1 & -1 & -1 & 23.08 & 304.06 & -103.86 & -44.9 \\
\hline 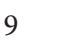 & -1 & -1 & -1 & +1 & -1 & -1 & 9.98 & 2.93 & -76.7 & -35.97 \\
\hline & +1 & -1 & -1 & +1 & -1 & -1 & 10.91 & 34.76 & -79.69 & -30.63 \\
\hline 1 & -1 & +1 & -1 & +1 & -1 & -1 & 3.94 & 17.13 & - & -37.45 \\
\hline 2 & +1 & +1 & -1 & +1 & -1 & -1 & 11.37 & 61.45 & -87.63 & -35.91 \\
\hline 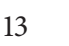 & -1 & -1 & +1 & +1 & -1 & -1 & 17.28 & 184.2 & -65.01 & -37.2 \\
\hline$T$ & +1 & -1 & +1 & +1 & -1 & & 2102 & 249.76 & -64.29 & -41.21 \\
\hline & -1 & +1 & +1 & +1 & -1 & -1 & 7.8 & 06.6 & -79.89 & -42.43 \\
\hline & +1 & +1 & +1 & +1 & -1 & -1 & 22.32 & 281.47 & -82.59 & -43.14 \\
\hline & -1 & -1 & -1 & -1 & +1 & & & & 48.17 & -18.59 \\
\hline & +1 & -1 & -1 & -1 & +1 & -1 & 0.73 & 63.33 & 57.78 & -17.65 \\
\hline o & -1 & +1 & -1 & -1 & +1 & -1 & 9.97 & 47.31 & 20.62 & -19.76 \\
\hline 20 & +1 & +1 & -1 & -1 & +1 & & 1100 & 91.35 & 20.14 & 2071 \\
\hline 21 & -1 & -1 & +1 & -1 & +1 & -1 & .29 & 266.17 & 67.45 & -35.37 \\
\hline 2 & +1 & -1 & +1 & -1 & +1 & -1 & 23.98 & 340.57 & 62.65 & -34.25 \\
\hline 23 & -1 & +1 & +1 & -1 & +1 & -1 & $100 ?$ & 258.73 & 29.44 & -36.02 \\
\hline 24 & +1 & +1 & +1 & -1 & +1 & -1 & 2.43 & 314.95 & 98 & -37.2 \\
\hline 25 & -1 & -1 & -1 & +1 & +1 & -1 & 81 & 12.57 & 53.05 & -22.82 \\
\hline 20 & +1 & -1 & -1 & +1 & +1 & -1 & 10.67 & 44.08 & 50.9 & -20.57 \\
\hline 27 & -1 & +1 & -1 & +1 & +1 & -1 & & 0.17 & 41.37 & -23.21 \\
\hline 28 & +1 & +1 & -1 & +1 & +1 & -1 & 1 & & 12 & 3.04 \\
\hline 29 & -1 & -1 & +1 & +1 & +1 & -1 & 1727 & 201.54 & 7100 & -52.71 \\
\hline 0 & +1 & -1 & +1 & +1 & +1 & -1 & 1.97 & 277.52 & 79.47 & -40.39 \\
\hline 31 & -1 & +1 & +1 & +1 & +1 & -1 & 18.04 & 28 & & -44.78 \\
\hline & +1 & +1 & +1 & +1 & +1 & -1 & & 288.89 & 52664 & -41.63 \\
\hline 33 & -1 & -1 & -1 & -1 & -1 & +1 & 10.01 & 18.39 & & -49.93 \\
\hline 4 & +1 & -1 & -1 & -1 & -1 & +1 & 11.05 & 55.37 & -65.77 & -35.69 \\
\hline & -1 & +1 & -1 & -1 & -1 & +1 & 10.3 & 39.32 & -84.83 & -63.8 \\
\hline 36 & +1 & +1 & -1 & -1 & -1 & +1 & & & -87.69 & -47.6 \\
\hline 37 & -1 & -1 & +1 & -1 & -1 & +1 & 17.82 & 213.16 & -51 & -31.01 \\
\hline 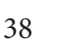 & +1 & -1 & +1 & -1 & -1 & +1 & 21.54 & 276.05 & -45.24 & -32.7 \\
\hline 39 & -1 & +1 & +1 & -1 & -1 & +1 & 19.11 & 242.5 & -19.4 & -36.66 \\
\hline 40 & +1 & +1 & +1 & -1 & -1 & +1 & 22.28 & 294.1 & -81.95 & -37.36 \\
\hline 11 & -1 & -1 & -1 & +1 & -1 & +1 & 10.07 & 8.96 & -56.66 & -41.44 \\
\hline 42 & +1 & -1 & -1 & +1 & -1 & +1 & 10.52 & 25.32 & -43.47 & -34.98 \\
\hline 43 & -1 & +1 & -1 & +1 & -1 & +1 & 10.17 & 25.25 & -62.55 & -50.4 \\
\hline 44 & +1 & +1 & -1 & +1 & -1 & +1 & 11.33 & 65.66 & -68.33 & -45.6 \\
\hline & -1 & -1 & +1 & +1 & -1 & +1 & 17.62 & 195.45 & -39.74 & -30.14 \\
\hline
\end{tabular}

TABle 5: Continued.

\begin{tabular}{lccccccccccc}
\hline Run & $w_{1}$ & $d_{1}$ & $t_{1}$ & $d_{2}$ & $t_{2}$ & $w_{2}$ & $R_{\mathrm{re}}(\Omega)$ & $X_{\mathrm{re}}(\Omega)$ & $X_{\mathrm{sc}}(\Omega)$ & $\left|S_{21}\right|(\mathrm{dB})$ \\
\hline 46 & +1 & -1 & +1 & +1 & -1 & +1 & 20.96 & 250.01 & -41.8 & -32.97 \\
47 & -1 & +1 & +1 & +1 & -1 & +1 & 18.43 & 222.91 & -57.19 & -35.37 \\
48 & +1 & +1 & +1 & +1 & -1 & +1 & 21.93 & 280.51 & -61.69 & -36.86 \\
49 & -1 & -1 & -1 & -1 & +1 & +1 & 10.17 & 49.11 & 79.06 & -24.02 \\
50 & +1 & -1 & -1 & -1 & +1 & +1 & 11.19 & 82.77 & 95.26 & -23.1 \\
51 & -1 & +1 & -1 & -1 & +1 & +1 & 10.04 & 53.62 & 58.22 & -25.16 \\
52 & +1 & +1 & -1 & -1 & +1 & +1 & 11.3 & 93.46 & 57.24 & -25.26 \\
53 & -1 & -1 & +1 & -1 & +1 & +1 & 19.48 & 276.21 & 109.07 & -68.59 \\
54 & +1 & -1 & +1 & -1 & +1 & +1 & 24.24 & 350.33 & 119.31 & -50.94 \\
55 & -1 & +1 & +1 & -1 & +1 & +1 & 17.67 & 240.59 & 67.23 & -53.59 \\
56 & +1 & +1 & +1 & -1 & +1 & +1 & 22.14 & 315.04 & 80.15 & -55.5 \\
57 & -1 & -1 & -1 & +1 & +1 & +1 & 9.92 & 18.08 & 90.26 & -27.86 \\
58 & +1 & -1 & -1 & +1 & +1 & +1 & 10.67 & 47.29 & 91.94 & -25.21 \\
59 & -1 & +1 & -1 & +1 & +1 & +1 & 9.73 & 31.43 & 67.56 & -27.87 \\
60 & +1 & +1 & -1 & +1 & +1 & +1 & 10.4 & 54.17 & 84.71 & -26.77 \\
61 & -1 & -1 & +1 & +1 & +1 & +1 & 17.41 & 206.82 & 121.66 & -45.6 \\
62 & +1 & -1 & +1 & +1 & +1 & +1 & 21.28 & 271.39 & 123.92 & -56.05 \\
63 & -1 & +1 & +1 & +1 & +1 & +1 & 17.97 & 229.22 & 79.09 & -49.58 \\
64 & +1 & +1 & +1 & +1 & +1 & +1 & 20.38 & 272.5 & 76.67 & -62.57 \\
\hline & & & & & & & & & &
\end{tabular}

defining relation for this design is $I=w_{1} \times d_{1} \times t_{1} \times t_{2}=$ $w_{1} \times w_{2} \times d_{2} \times t_{2}=d_{1} \times d_{2} \times w_{2} \times t_{1}$, and the complex aliasing patterns of effects are identified by

$$
\begin{gathered}
w_{1}+d_{1} * t_{1} * t_{2}+d_{2} * t_{2} * w_{2}+w_{1} * d_{1} * t_{1} * d_{2} * w_{2} \\
d_{1}+w_{1} * t_{1} * t_{2}+t_{1} * d_{2} * w_{2}+w_{1} * d_{1} * d_{2} * t_{2} * w_{2} \\
t_{1}+w_{1} * d_{1} * t_{2}+d_{1} * d_{2} * w_{2}+w_{1} * t_{1} * d_{2} * t_{2} * w_{2} \\
d_{2}+w_{1} * t_{2} * w_{2}+d_{1} * t_{1} * w_{2}+w_{1} * d_{1} * t_{1} * d_{2} * t_{2} \\
t_{2}+w_{1} * d_{1} * t_{1}+w_{1} * d_{2} * w_{2}+d_{1} * t_{1} * d_{2} * t_{2} * w_{2} \\
w_{2}+w_{1} * d_{2} * t_{2}+d_{1} * t_{1} * d_{2}+w_{1} * d_{1} * t_{1} * t_{2} * w_{2} \\
w_{1} * d_{1}+t_{1} * t_{2}+w_{1} * t_{1} * d_{2} * w_{2}+d_{1} * d_{2} * t_{2} * w_{2} \\
w_{1} * t_{1}+d_{1} * t_{2}+w_{1} * d_{1} * d_{2} * w_{2}+t_{1} * d_{2} * t_{2} * w_{2} \\
w_{1} * d_{2}+t_{2} * w_{2}+w_{1} * d_{1} * t_{1} * w_{2}+d_{1} * t_{1} * d_{2} * t_{2} \\
w_{1} * t_{2}+d_{1} * t_{1}+d_{2} * w_{2}+w_{1} * d_{1} * t_{1} * d_{2} * t_{2} * w_{2} \\
w_{1} * w_{2}+d_{2} * t_{2}+w_{1} * d_{1} * t_{1} * d_{2}+d_{1} * t_{1} * t_{2} * w_{2} \\
d_{1} * d_{2}+t_{1} * w_{2}+w_{1} * d_{1} * t_{2} * w_{2}+w_{1} * t_{1} * d_{2} * t_{2} \\
d_{1} * w_{2}+t_{1} * d_{2}+w_{1} * d_{1} * d_{2} * t_{2}+w_{1} * t_{1} * t_{2} * w_{2} \\
w_{1} * d_{1} * d_{2}+w_{1} * t_{1} * w_{2}+d_{1} * t_{2} * w_{2}+t_{1} * d_{2} * t_{2} \\
w_{1} * d_{1} * w_{2}+w_{1} * t_{1} * d_{2}+d_{1} * d_{2} * t_{2}+t_{1} * t_{2} * w_{2} \\
I+w_{1} * d_{1} * t_{1} * t_{2}+w_{1} * w_{2} * d_{2} * t_{2}+d_{1} * d_{2} * w_{2} * t_{1}
\end{gathered}
$$

Once again, $w_{1}+d_{1} * t_{1} * t_{2}+d_{2} * t_{2} * w_{2}+w_{1} *$ $d_{1} * t_{1} * d_{2} * w_{2}$ indicates that the main effect of $w_{1}$, the three-factor interaction of $d_{1}, t_{1}$, and $t_{2}$, the three-factor interaction of $d_{2}, t_{2}$, and $w_{2}$, and the five-factor interaction of $w_{1}, d_{1}, t_{1}, d_{2}$, and $w_{2}$ are confounded together and so forth. The resultant simulation combination and the corresponding results are shown in Table 6 . Based on the effect calculation, 
16 estimated effects for the above confounding structures are thus obtained. Omitting the higher-order interactions, the response surface models with significant coefficients are

$$
\begin{aligned}
& \widehat{R}_{\mathrm{re}}(\Omega)=15.23+0.99\left(\frac{w_{1}-3.75}{0.25}\right) \\
& +4.68\left(\frac{t_{1}-3.25}{0.25}\right)-0.35\left(\frac{d_{2}-1}{0.2}\right) \\
& +0.45\left(\frac{w_{1}-3.75}{0.25}\right)\left(\frac{t_{1}-3.25}{0.25}\right) \text {, } \\
& \widehat{X}_{\mathrm{re}}(\Omega)=150.35+20.18\left(\frac{w_{1}-3.75}{0.25}\right) \\
& +105.30\left(\frac{t_{1}-3.25}{0.25}\right) \\
& -14.02\left(\frac{d_{2}-1}{0.2}\right) \\
& \widehat{X}_{\mathrm{sc}}(\Omega)=-4.51-12.96\left(\frac{d_{1}-1}{0.2}\right) \\
& +5.45\left(\frac{t_{1}-3.25}{0.25}\right)+6.53\left(\frac{d_{2}-1}{0.2}\right) \\
& +71.53\left(\frac{t_{2}-3}{0.25}\right) \\
& +15.65\left(\frac{w_{2}-2.75}{0.25}\right) \text {, } \\
& \left|\widehat{S}_{21}\right|(\mathrm{dB})=-39.10-8.01\left(\frac{t_{1}-3.25}{0.25}\right) \\
& -3.23\left(\frac{w_{2}-2.75}{0.25}\right) \\
& -8.22\left(\frac{t_{1}-3.25}{0.25}\right)\left(\frac{t_{2}-3}{0.25}\right) \\
& -3.11\left(\frac{t_{2}-3}{0.25}\right)\left(\frac{w_{2}-2.75}{0.25}\right) \text {. }
\end{aligned}
$$

Following the same procedure of numerical optimization, the optimum parameters are $w_{1}=3.78 \mathrm{~mm}, d_{1}=0.95 \mathrm{~mm}$, $t_{1}=3.18 \mathrm{~mm}, w_{2}=3 \mathrm{~mm}, d_{2}=1.18 \mathrm{~mm}$, and $t_{2}=2.98 \mathrm{~mm}$. This design is then verified by HFSS, and the simulated results at $915 \mathrm{MHz}$ are $R_{\mathrm{re}}=13.32 \Omega, X_{\mathrm{re}}=110.63 \Omega, X_{\mathrm{sc}}=0.72 \Omega$, and $\left|S_{21}\right|=-50.43 \mathrm{~dB}$. The simulated results show that the impedance requirements are met, and the mutual coupling is kept at a very low level. In comparison with the results obtained via the full factorial design, the performances determined by orthogonal fractional experiments are impressive, yet the number of required simulations is reduced by a factor of four.

In order to examine the detailed performances of the proposed method, the optimum structure was fabricated and tested. The return loss of the receiving antenna with the backscattering antenna being open-circuited and the isolation between the two antennas are shown in Figure 5,
TABLE 6: The $2^{6-2}$ orthogonal fractional experiment and the simulated results (at $915 \mathrm{MHz}$ ).

\begin{tabular}{lcccccccccc}
\hline Run & $w_{1}$ & $d_{1}$ & $t_{1}$ & $d_{2}$ & $t_{2}$ & $w_{2}$ & $R_{\mathrm{re}}(\Omega)$ & $X_{\mathrm{re}}(\Omega)$ & $X_{\mathrm{sc}}(\Omega)$ & $\left|S_{21}\right|(\mathrm{dB})$ \\
\hline 1 & -1 & -1 & -1 & -1 & -1 & -1 & 10.18 & 20.64 & -96.97 & -32.56 \\
2 & +1 & -1 & -1 & -1 & +1 & -1 & 10.75 & 63.33 & 57.78 & -17.65 \\
3 & -1 & +1 & -1 & -1 & +1 & +1 & 10.04 & 53.62 & 58.22 & -25.16 \\
4 & +1 & +1 & -1 & -1 & -1 & +1 & 11.54 & 78.88 & -87.69 & -47.6 \\
5 & -1 & -1 & +1 & -1 & +1 & +1 & 19.48 & 276.21 & 109.07 & -68.59 \\
6 & +1 & -1 & +1 & -1 & -1 & +1 & 21.54 & 276.05 & -45.24 & -32.7 \\
7 & -1 & +1 & +1 & -1 & -1 & -1 & 18.66 & 231.22 & -112.58 & -46.48 \\
8 & +1 & +1 & +1 & -1 & +1 & -1 & 22.43 & 314.95 & 29.08 & -37.2 \\
9 & -1 & -1 & -1 & +1 & -1 & +1 & 10.07 & 8.96 & -56.66 & -41.44 \\
10 & +1 & -1 & -1 & +1 & +1 & +1 & 10.67 & 47.29 & 91.94 & -25.21 \\
11 & -1 & +1 & -1 & +1 & +1 & -1 & 9.77 & 26.17 & 41.37 & -23.21 \\
12 & +1 & +1 & -1 & +1 & -1 & -1 & 11.37 & 61.45 & -87.63 & -35.91 \\
13 & -1 & -1 & +1 & +1 & +1 & -1 & 17.27 & 201.54 & 71.99 & -52.71 \\
14 & +1 & -1 & +1 & +1 & -1 & -1 & 21.02 & 249.76 & -64.29 & -41.21 \\
15 & -1 & +1 & +1 & +1 & -1 & +1 & 18.43 & 222.91 & -57.19 & -35.37 \\
16 & +1 & +1 & +1 & +1 & +1 & +1 & 20.38 & 272.5 & 76.67 & -62.57 \\
\hline
\end{tabular}

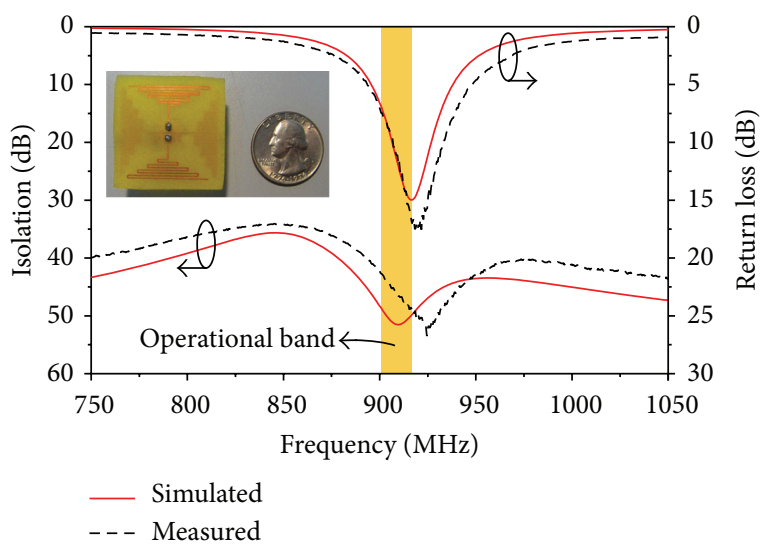

FIGURE 5: The return loss of the receiving antenna with the backscattering antenna being open-circuited and the isolation between the two antennas. Shown in the inset is the photograph of the fabricated prototype.

which clearly indicates that the bandwidth requirement is satisfied and the isolations are all greater than $43 \mathrm{~dB}$. More importantly, the optimization efficiency is greatly enhanced; only 16 simulations are required, yet the performances attained are still excellent.

\section{A Dual-Band Internal Antenna for LTE Handheld Devices}

After describing these successful experiences in RFID applications, the technique of orthogonal fractional experiments is examined through an extremely complicated example. Figure 6 depicts the design environment of an internal antenna for LTE handheld devices [21]. The antenna structure is so complex that 12 design parameters, including the lengths 


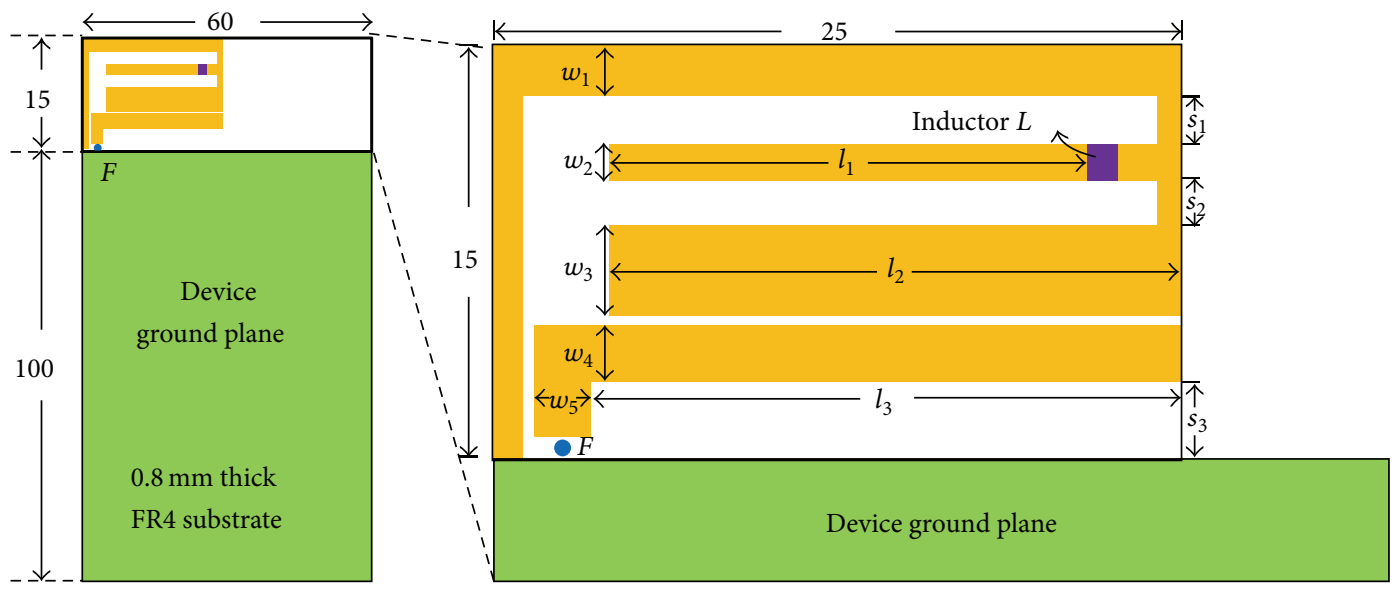

FIGURE 6: The benchmarking configuration of the internal antenna for LTE handheld devices.

TABLE 7: Ranges for the design parameter of the internal antenna for LTE handheld devices.

\begin{tabular}{lcc}
\hline Parameter & Low level $(-1)$ & High level $(+1)$ \\
\hline$L$ & $30 \mathrm{nH}$ & $40 \mathrm{nH}$ \\
$w_{1}$ & $1.5 \mathrm{~mm}$ & $2.0 \mathrm{~mm}$ \\
$w_{2}$ & $0.8 \mathrm{~mm}$ & $1.2 \mathrm{~mm}$ \\
$w_{3}$ & $2.5 \mathrm{~mm}$ & $3.5 \mathrm{~mm}$ \\
$w_{4}$ & $1.0 \mathrm{~mm}$ & $2.0 \mathrm{~mm}$ \\
$w_{5}$ & $1.0 \mathrm{~mm}$ & $2.0 \mathrm{~mm}$ \\
$s_{1}$ & $2.8 \mathrm{~mm}$ & $3.3 \mathrm{~mm}$ \\
$s_{2}$ & $0.4 \mathrm{~mm}$ & $0.6 \mathrm{~mm}$ \\
$s_{3}$ & $2.3 \mathrm{~mm}$ & $2.8 \mathrm{~mm}$ \\
$l_{1}$ & $18 \mathrm{~mm}$ & $20.5 \mathrm{~mm}$ \\
$l_{2}$ & $22 \mathrm{~mm}$ & $23.5 \mathrm{~mm}$ \\
$l_{3}$ & $20 \mathrm{~mm}$ & $21.5 \mathrm{~mm}$ \\
\hline
\end{tabular}

of the segments $l_{1}, l_{2}, l_{3}, w_{1}, w_{2}, w_{3}, w_{4}$, and $w_{5}$, the spacing between the segments $s_{1}, s_{2}$, and $s_{3}$, and the inductance of the chip inductor $L$, must be considered into the design process. The design objective is to generate a dual-band LTE operation covering the communication bands of LTE700, GSM1800, GSM1900, UMTS, LTE2300, and LTE2500, namely, 698$806 \mathrm{MHz}$ and 1710-2690 MHz. In this case, the design cycle will be greatly expanded if trial-and-error approaches or onefactor-at-a-time parametric studies are adopted. Meanwhile, performing full factorial designs is improper because a $2^{12}$ full experiment requires 4,048 full-wave simulations, which are too costly to meet the radical innovation of mobile phones.

To reduce the number of simulations, a $2^{12-7}$ orthogonal fractional experiment is performed to optimize the dualband impedance characteristics. The response variables are the lowest frequency that has a $6-\mathrm{dB}$ return loss $f_{6-\mathrm{dB}}$ and the bandwidth within the lower band (698-806 MHz) $\Delta f$. The levels of the 12 design parameters are listed in Table 7. Next, $L, w_{1}, w_{2}, w_{3}$, and $w_{4}$ are selected as the basic variables, and their associated $2^{5}$ full factorial design is constructed. The states of the 7 additional variables, namely, $w_{5}, s_{1}, s_{2}$, $s_{3}, l_{1}, l_{2}$, and $l_{3}$, are assigned by $w_{5}=L \times w_{1} \times w_{2}, s_{1}=$ $L \times w_{2} \times w_{3}, s_{2}=w_{1} \times w_{2} \times w_{3}, s_{3}=L \times w_{1} \times w_{3}$, $l_{1}=L \times w_{3} \times w_{4}, l_{2}=L \times w_{2} \times w_{4}$, and $l_{3}=L \times$ $w_{1} \times w_{4}$. According to the theory of optimum experimental designs, the proposed design generators are proved to be the minimum aberration design among all the resolution IV experiments with 32 runs, so the important main effects of all the design parameters are estimated without confounding with each other. After simulating the value of the response variables, the response surface models with the significant coefficients can be determined; hence, the design problem can be modeled into a constrained programming problem, in which $\Delta f$ should be maximized subject to the constraint of $688 \leq f_{6-\mathrm{dB}} \leq 708$. It is therefore straightforward to determine the optimum solution, the parameters of which are $l_{1}=20.0 \mathrm{~mm}, l_{2}=23.0 \mathrm{~mm}, l_{3}=21.5 \mathrm{~mm}, w_{1}=1.8 \mathrm{~mm}$, $w_{2}=1.0 \mathrm{~mm}, w_{3}=3.2 \mathrm{~mm}, w_{4}=1.5 \mathrm{~mm}, w_{5}=2.0 \mathrm{~mm}$, $s_{1}=3.3 \mathrm{~mm}, s_{2}=0.5 \mathrm{~mm}, s_{3}=2.8 \mathrm{~mm}$, and $L=36 \mathrm{nH}$.

In order to facilitate assessing the efficiency of orthogonal fractional experiments, another reference antenna obtained by $2^{12}$ full factorial design of experiment was developed. That is, the reference antenna was also constructed by the DOE procedure, but all the significant higher-order interactions are incorporated into the response surface models. The resultant parameters derived from these models are $l_{1}=20.5 \mathrm{~mm}$, $l_{2}=23.5 \mathrm{~mm}, l_{3}=21.3 \mathrm{~mm}, w_{1}=2.0 \mathrm{~mm}, w_{2}=0.8 \mathrm{~mm}$, $w_{3}=3.1 \mathrm{~mm}, w_{4}=1.2 \mathrm{~mm}, w_{5}=1.8 \mathrm{~mm}, s_{1}=3.2 \mathrm{~mm}$, $s_{2}=0.4 \mathrm{~mm}, s_{3}=2.8 \mathrm{~mm}$, and $L=34 \mathrm{nH}$. Accordingly, the simulated reflection coefficients of the two approaches are shown and compared in Figure 7. Clearly, both experimental strategies cover the desired bandwidth by $3: 1$ VSWR, but the technique of orthogonal fractional experiments reduces the design effort by $99.2 \%$. In addition, an interesting finding is that the two optimization approaches have agreeable performances in the lower band $(698-806 \mathrm{MHz})$ yet the upper band (1710-2690 MHz) has a frequency shift. This is because the selected response variables only target at the return loss in the lower band. Since the bandwidth in the upper band can be readily obtained by the driven element, only the characteristics of the lower bandwidth, namely, $f_{6-\mathrm{dB}}$ 


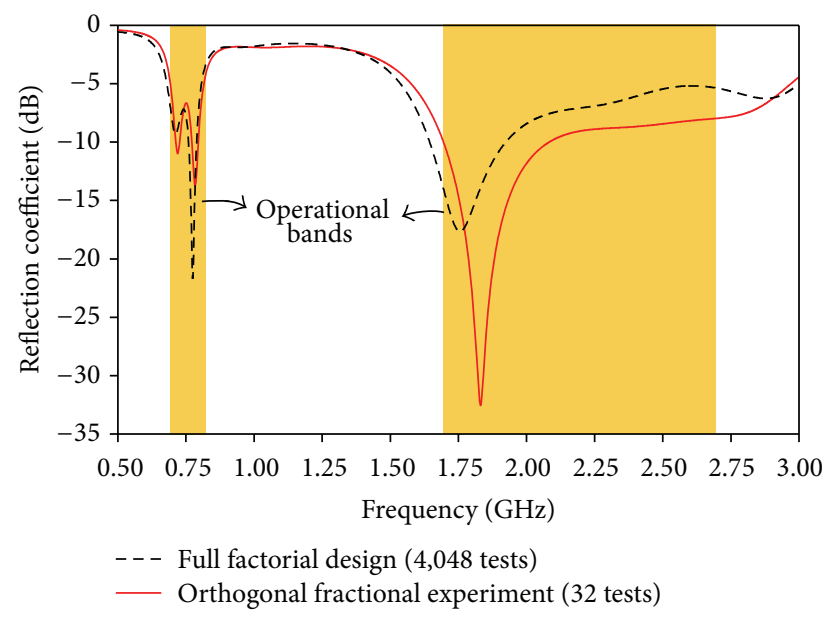

FIGURE 7: Comparison of the optimized internal antennas between the full factorial design and the orthogonal fractional experiment.

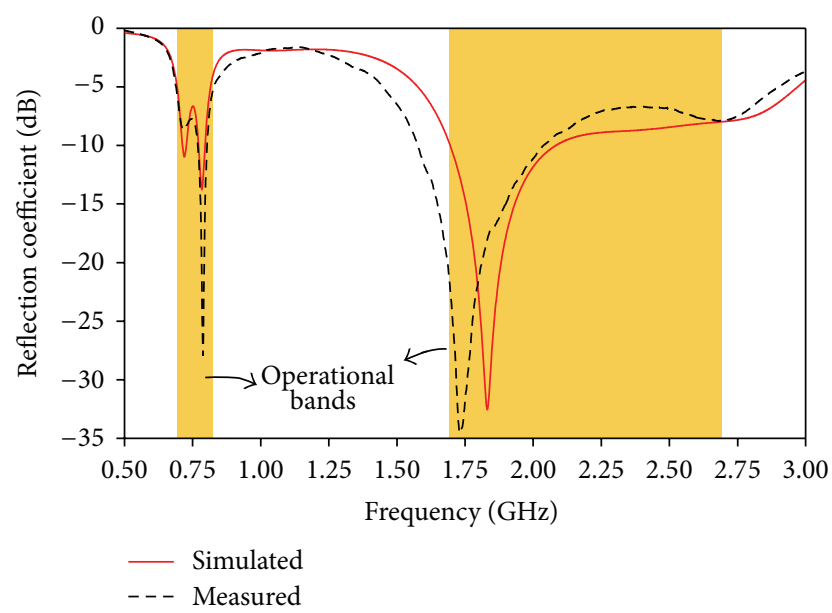

FIGURE 8: Simulated and measured reflection coefficients of the optimized antenna structure.

and $\Delta f$, are assigned as the response variables. As a result, both the optimization approaches focus on the impedance bandwidth in the lower band, so they found comparable performances in 698-806 MHz. However, the effects of the optimum parameters on the upper band are not cast into the optimization process, so the antenna structure determined by the full factorial design and the configuration obtained via the orthogonal fractional experiment have divergent performances in the upper band.

For verification purpose, the optimum antenna obtained by orthogonal fractional experiments was also fabricated and tested. The simulated and measured reflection coefficients of the optimized antenna are shown in Figure 8. They exhibit very good agreement, and both the lower band $(698-806 \mathrm{MHz})$ and higher band (1710-2690 MHz) meet the bandwidth requirement. Besides, the shift appearing in the upper band may result from the fabrication error. While the lower band is worked by uniting two resonance modes, the bandwidth in the upper band is attained by

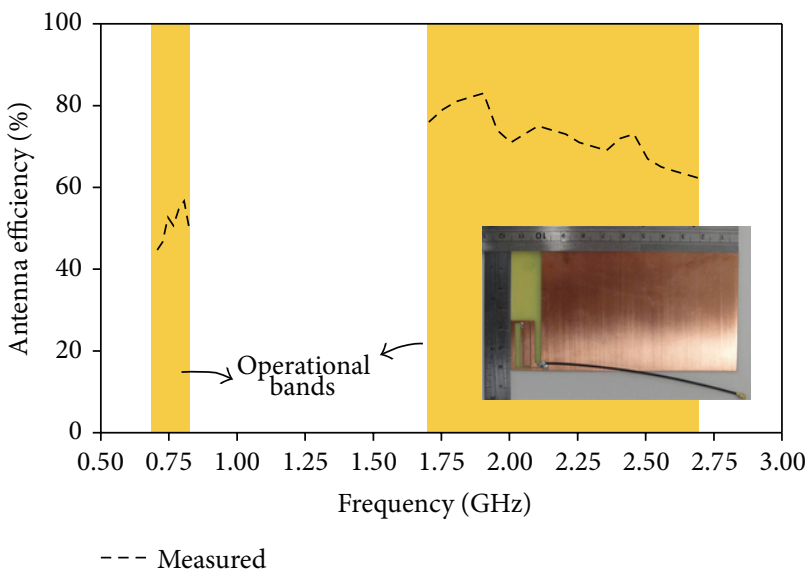

FIGURE 9: Measured antenna efficiencies of the optimized antenna structure. Shown in the inset is the photograph of the fabricated prototype.

only one resonance mode resulting from the driven strip. It is speculated that the single resonance mode has a larger variance in the fabrication process, so the upper band has a larger frequency shift as compared to the lower band.

Meanwhile, the measured antenna efficiencies are shown in Figure 9. The level of the efficiencies is larger than $40 \%$, so the optimized antenna is very suitable for LTE handheld devices. Most significantly, by applying the technique of orthogonal fractional experiments, such a complex structure including 12 design parameters is determined with merely 32 simulations. In sum, orthogonal fractional experiments greatly reduce the amount of simulations, thereby leading to a shorter design cycle and a lower development cost.

\section{Conclusion}

This paper validates the efficiency of orthogonal fractional experiments for design of complex antenna structures. Unlike nature-inspired heuristic algorithms, which extensively search the entire solution space, the technique of orthogonal fractional experiments builds up the response surface models via performing predefined simulation combination. Moreover, this paper shows that, by performing a small fraction of full factorial experiment, the antenna structure can be successfully developed even though the higher-order interactions are omitted.

Several design problems have been addressed by the proposed technique. The associated results show that orthogonal fractional experiments have great competence in efficiency and accuracy. Specifically, an inductive-feed meander dipole for RFID tags is successfully designed with merely 8 tests. In addition, a complex dual-antenna system for passive RFID tags is developed by performing simply 16 simulations; although the design space is limited to $0.1 \times$ $0.1 \lambda^{2}$, the proposed method obtains a design with very low mutual coupling between the constituent antennas (isolation $>43 \mathrm{~dB}$ throughout the operational band) while the requirement of antenna impedance is fulfilled. Furthermore, an 
internal antenna for LTE dual-band applications occupying $25 \times 15 \mathrm{~mm}^{2}$ is successfully designed by merely 32 simulations; accordingly, orthogonal fractional experiments reduce the design effort from full factorial designs by $99.2 \%$. These results indicate that the proposed technique can achieve substantial time savings and lead to a shorter design cycle.

\section{Conflict of Interests}

The authors declare that there is no conflict of interests regarding the publication of this paper.

\section{Acknowledgment}

This work was supported by the Ministry of Science and Technology, Taiwan, under Contract MOST 103-2221-E-027004.

\section{References}

[1] J. M. Johnson and Y. Rahmat-Samii, "Genetic algorithms and method of moments (GA/MOM) for the design of integrated antennas," IEEE Transactions on Antennas and Propagation, vol. 47, no. 10, pp. 1606-1614, 1999.

[2] J. M. Jayasinghe and D. Uduwawala, "A novel multiband miniature planar inverted $\mathrm{F}$ antenna design for bluetooth and WLAN applications," International Journal of Antennas and Propagation, vol. 2015, Article ID 970152, 6 pages, 2015.

[3] C.-W. Liu and C.-C. Chen, "A UWB three-layer dielectric rod antenna with constant gain, pattern and phase center," IEEE Transactions on Antennas and Propagation, vol. 60, no. 10, pp. 4500-4508, 2012.

[4] N. T. Tokan, "Optimization of the UWB feed antenna position in reflector applications," International Journal of Antennas and Propagation, vol. 2014, Article ID 961818, 7 pages, 2014.

[5] Y.-L. Li, W. Shao, L. You, and B.-Z. Wang, "An improved PSO algorithm and its application to UWB antenna design," IEEE Antennas and Wireless Propagation Letters, vol. 12, pp. 12361239, 2013.

[6] M. Zubair and M. Moinuddin, "Joint optimization of microstrip patch antennas using particle swarm optimization for UWB systems," International Journal of Antennas and Propagation, vol. 2013, Article ID 649049, 8 pages, 2013.

[7] L. Lizzi, F. Viani, R. Azaro, and A. Massa, "Optimization of a spline-shaped UWB antenna by PSO," IEEE Antennas and Wireless Propagation Letters, vol. 6, pp. 182-185, 2007.

[8] C. Yu, T. Xu, and C. Liu, "Design of a novel UWB omnidirectional antenna using particle swarm optimization," International Journal of Antennas and Propagation, vol. 2015, Article ID 303195, 7 pages, 2015.

[9] A. Chen, T. Jiang, Z. Chen, and Y. Zhang, "A genetic and simulated annealing combined algorithm for optimization of wideband antenna matching networks," International Journal of Antennas and Propagation, vol. 2012, Article ID 251624, 6 pages, 2012.

[10] E. Rajo-lglesias and Ó. Quevedo-Teruel, "Linear array synthesis using an ant-colony-optimization-based algorithm," IEEE Antennas and Propagation Magazine, vol. 49, no. 2, pp. 70-79, 2007.
[11] S. Karimkashi and A. A. Kishk, "Invasive weed optimization and its features in electromagnetics," IEEE Transactions on Antennas and Propagation, vol. 58, no. 4, pp. 1269-1278, 2010.

[12] K. Naishadham, "Design of experiments as a microwave cad tool," Microwave and Optical Technology Letters, vol. 52, no. 5, pp. 1020-1024, 2010.

[13] P. M. Watson and K. C. Gupta, "Design and optimization of CPW circuits using EM-ANN models for CPW components," IEEE Transactions on Microwave Theory and Techniques, vol. 45, no. 12, pp. 2515-2523, 1997.

[14] L. Yang, L. J. Martin, D. Staiculescu, C. P. Wong, and M. M. Tentzeris, "Conformal magnetic composite RFID for wearable $\mathrm{RF}$ and bio-monitoring applications," IEEE Transactions on Microwave Theory and Techniques, vol.56, no. 12, pp. 3223-3230, 2008.

[15] D. Staiculescu, N. Bushyager, A. Obatoyinbo, L. J. Martin, and M. M. Tentzeris, "Design and optimization of 3-D compact stripline and microstrip Bluetooth/WLAN balun architectures using the design of experiments technique," IEEE Transactions on Antennas and Propagation, vol. 53, no. 5, pp. 1805-1812, 2005.

[16] J. Maldonado-Vargas and R. A. Rodríguez-Solís, "Analysis of a cavity backed annular slot ring antenna using design of experiment techniques," in Proceedings of the IEEE Antennas and Propagation Society International Symposium and URSI Radio Science Meeting (APSURSI '14), pp. 1501-1502, IEEE, Memphis, Tenn, USA, July 2014.

[17] M. Hamada and N. Balakrishnan, "Analyzing unreplicated factorial experiments: a review with some new proposals," Statistica Sinica, vol. 8, no. 1, pp. 1-41, 1998.

[18] H.-W. Son and C.-S. Pyo, "Design of RFID tag antennas using an inductively coupled feed," Electronics Letters, vol. 41, no. 18, pp. 994-996, 2005.

[19] Impinj, "Monza 4 tag chip datasheet," http://www.impinj.com/.

[20] Y.-S. Chen, S.-Y. Chen, and H.-J. Li, "A novel dual-antenna structure for UHF RFID tags," IEEE Transactions on Antennas and Propagation, vol. 59, no. 11, pp. 3950-3960, 2011.

[21] Y. S. Chen and T. Y. Ku, "Development of a compact LTE dualband antenna using fractional factorial design," IEEE Antennas and Wireless Propagation Letters, vol. 14, pp. 1097-1100, 2015. 

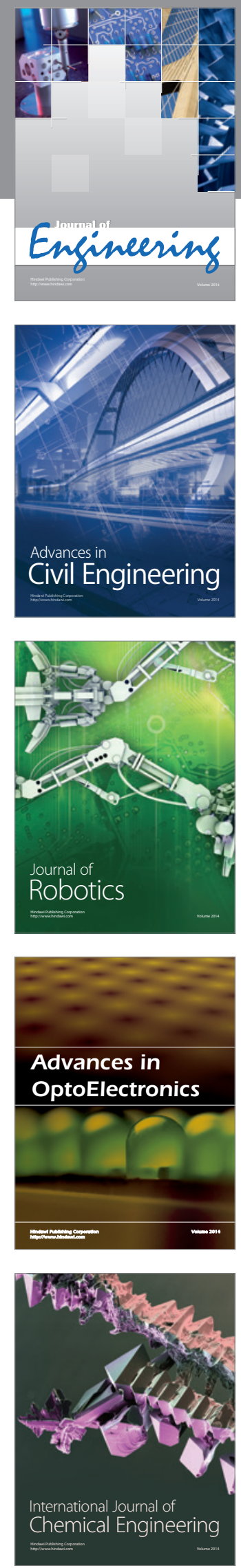

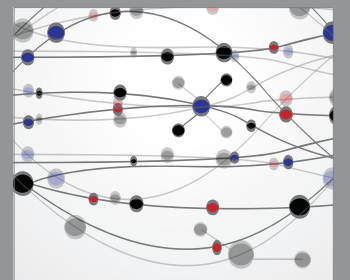

The Scientific World Journal
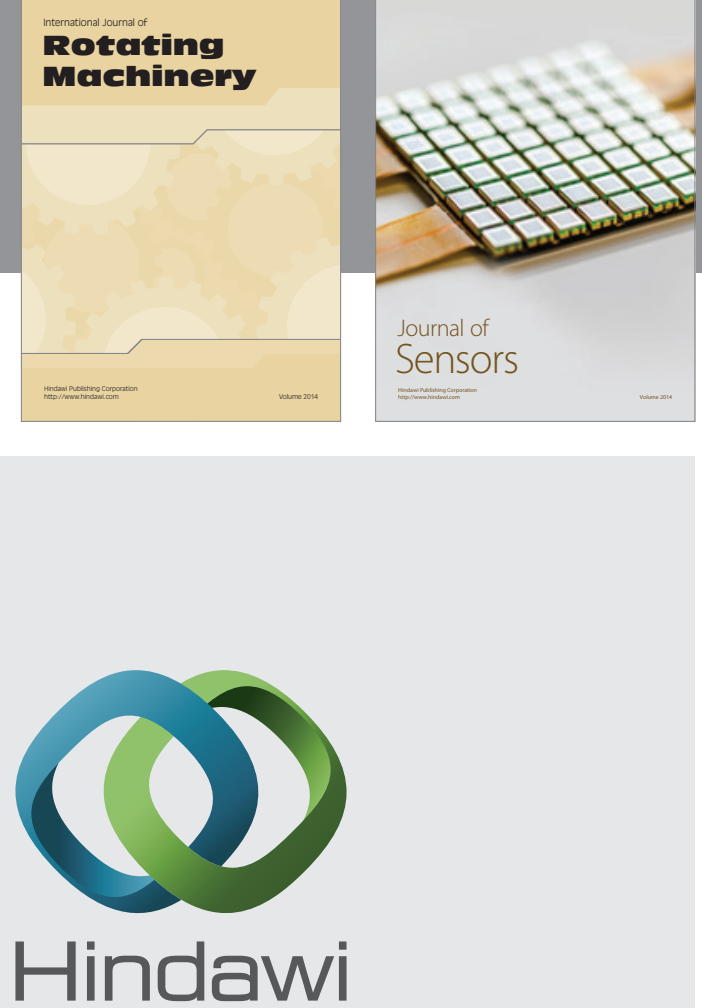

Submit your manuscripts at http://www.hindawi.com
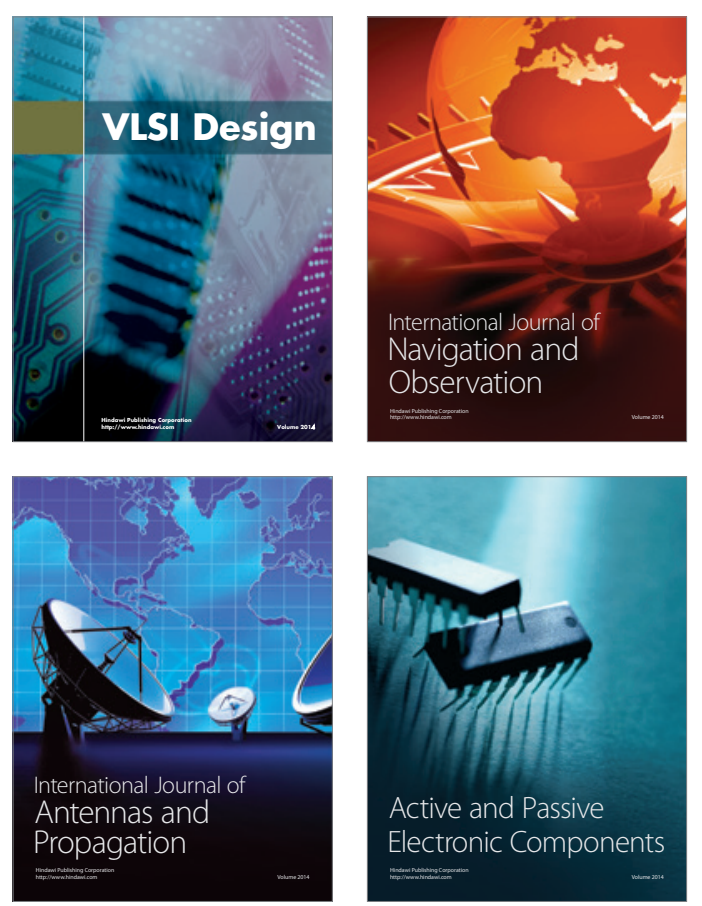
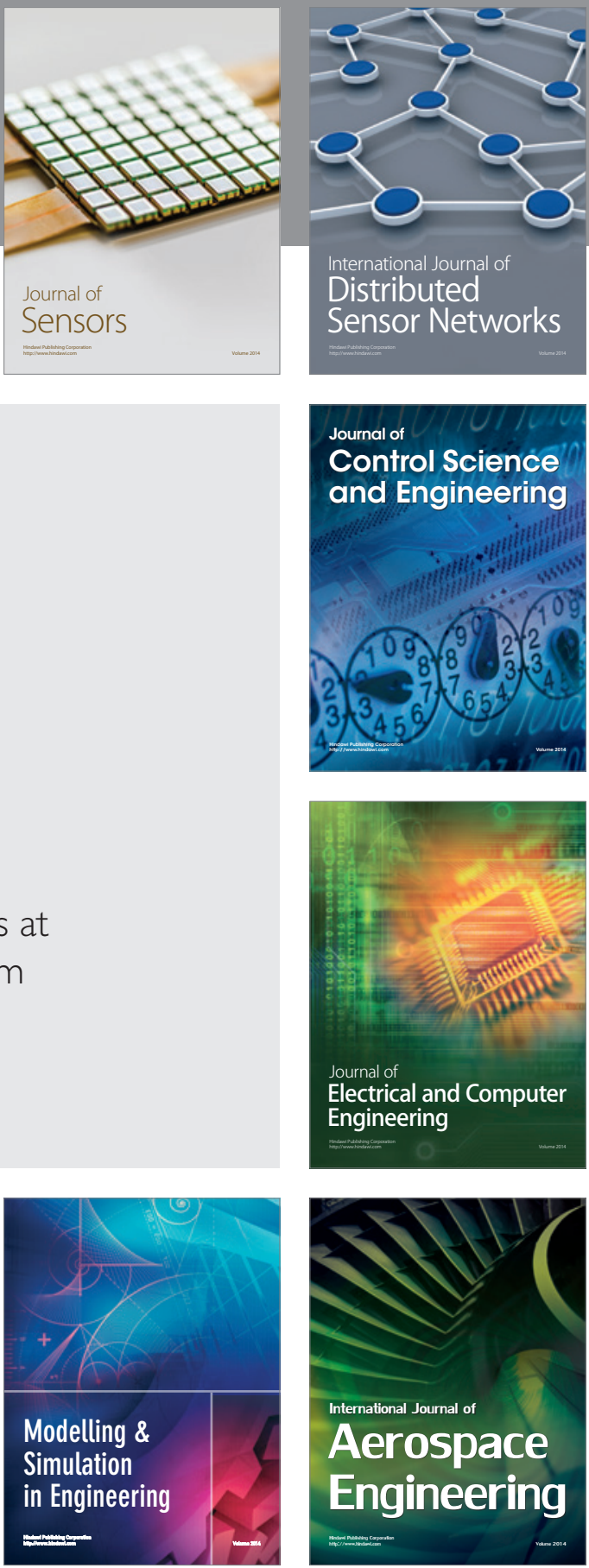

Journal of

Control Science

and Engineering
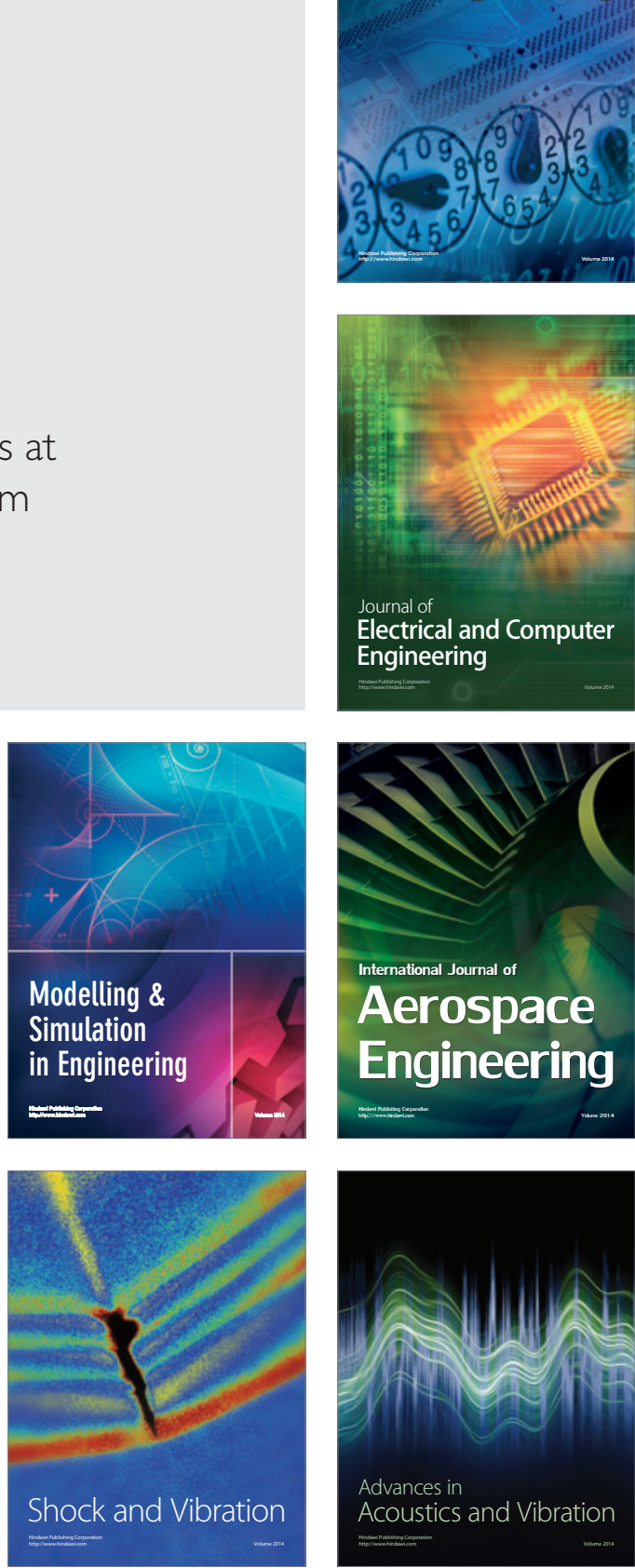